Revue d'histoire de l'Amérique française

REVUE D.HISTOIRE DE L'AMÉRIQUE FRANÇAISE

\title{
L'histoire au carrefour des sciences humaines
}

\section{Alfred Dubuc}

Volume 24, numéro 3, décembre 1970

URI : https://id.erudit.org/iderudit/302986ar

DOI : https://doi.org/10.7202/302986ar

Aller au sommaire du numéro

Éditeur(s)

Institut d'histoire de l'Amérique française

ISSN

0035-2357 (imprimé)

1492-1383 (numérique)

Découvrir la revue

Citer cet article

Dubuc, A. (1970). L'histoire au carrefour des sciences humaines. Revue

d'histoire de l'Amérique française, 24(3), 331-340.

https://doi.org/10.7202/302986ar d'utilisation que vous pouvez consulter en ligne.

https://apropos.erudit.org/fr/usagers/politique-dutilisation/ 


\title{
L'HISTOIRE AU CARREFOUR DES SCIENCES HUMAINES *
}

\author{
Alfred Dubuc \\ Université du Québec \\ (Montréal)
}

Depuis près d'un demi-siècle, déjà, les sciences sociales, en se développant, ont fécondé singulièrement l'histoire. En permettant à l'homme d'aujourd'hui de se mieux connaître, en développant des outils d'analyse sans cesse plus adéquats, ces nouvelles sciences ont fourni à l'histoire des interrogations nouvelles qui permettent à l'homme de prendre une meilleure conscience du mouvement qui l'entraîne et, par là, de nourrir des projets plus éclairés d'édification d'un monde meilleur.

Cet enrichissement récent de l'histoire au contact des sciences sociales s'est accompagné, je crois, d'un échange réciproque, l'histoire apportant aux sciences nouvelles une dimension et une problématique particulières qu'elle avait appris à développer depuis déjà fort longtemps. De toutes les sciences humaines, en effet, l'histoire peut prétendre, à juste titre, être la mère des autres. Vieille qu'elle est, en effet, d'une tradition plus de deux fois millénaire, qui lui a appris à s'interroger sans cesse sur l'homme, son évolution et sa destinée, l'histoire peut se féliciter d'avoir engendré les sciences sociales, ses filles prometteuses.

Certes, s'est-elle transformée depuis quelques décennies. A l'aide des sciences sociales, elle a étendu le champ de son enquête et affiné ses outils d'analyse.

J'aimerais, toutefois, soutenir qu'elle n'a pas cessé de jouer, dans la société humaine, à peu près le même rôle que celui qu'avaient jadis défini Hérodote et Thucydide. Peut-être même que, devant l'évolution la plus récente de certaines des sciences humaines, il devient opportun de proclamer, une fois encore, la fonction indispensable et inaliénable que remplit la démarche historique.

Je propose que, pour les fins de cette discussion, l'on distingue l'histoire comme connaissance, de l'histoire comme cul-

* Texte d'une communication présentée à Moscou, en août 1970, au $13^{e}$ Congrès du Comité international des sciences historiques, dans le cadre des travaux de la session de méthodologie (groupe sur l'historien et les sciences sociales).

[ 331 ]

RHAF, vol. 24, no 3 (décembre 1970) 
ture, quitte à réunir ensuite ces deux aspects pour analyser les relations réciproques qu'ils nourrissent entre eux. Dans une démarche, en effet, l'historien tente d'objectiver le passé. Dans une autre approche, fort différente, la connaissance acquise reflète sur le sujet connaissant, pénètre sa conscience et le transforme. Voilà les deux démarches intellectuelles qui se nourrissent l'une l'autre et qui, ensemble, l'une avec l'autre, l'une par l'autre, définissent indissolublement l'histoire. On conviendra facilement, toutefois, qu'elles sont, au niveau de l'épistémologie, fondamentalement différentes.

\section{De l'histoire comme connaissance}

Depuis l'époque où s'est formé l'esprit occidental et où l'homme a voulu réfléchir sur sa destinée, les historiens ont cherché à décrire le passé de leur société, s'efforçant de se placer en dehors de l'objet de leur recherche. Ils ont cherché à décrire le passé, non seulement pour le plaisir de le connaître, mais aussi pour le comprendre, pour l'expliquer. De toute antiquité, la connaissance du passé s'est inspiré de deux préoccupations différentes. Déjà celui qu'on considère comme le premier historien désirait, à la fois, présenter "l'exposé de l'enquête entreprise par Hérodote d'Halicarnasse pour empêcher que les actions accomplies par les hommes ne s'effacent avec le temps..." et, en même temps, découvrir "pour quelle cause Grecs et Perses barbares se combattirent..."

Thucydide, quant à lui, proclamait déjà l'obligation pour l'historien de vérifier l'authenticité des documents et la crédibilité de leurs auteurs. Il croyait, en outre, à l'existence de lois humaines dont la connaissance permettrait de prévoir l'avenir. "Quant aux événements de la guerre, affirmait-il, je n'ai pas jugé bon de les rapporter sur la loi du premier venu, ni d'après mon opinion... Cette recherche n'allait pas sans peine, parce que ceux qui ont assisté aux événements ne les rapportaient pas de la même manière et parlaient selon les intérêts de leur parti ou selon leurs souvenirs variables... Il me suffira que ceux qui veulent voir clair dans les faits analogues que l'avenir, selon la loi des choses humaines, ne peut manquer de ramener, jugent utile mon histoire".

Appelons, si vous y consentez, de façon imparfaite, j'en conviens, mais valable pour mon dessein, histoire positive, "l'enquête" qui veut retrouver les faits du passé pour eux-mêmes, et histoire compréhensive, la démarche par laquelle l'humanité interroge sans cesse son passé de façon à le mieux comprendre et, 
ultimement, à mieux expliquer le présent, sinon à prévoir l'avenir.

Je vous propose de reconnaître que depuis qu'elle existe, l'histoire a été entraînée dans un mouvement de pendule, recherchant pendant quelque temps la connaissance exacte de son passé, puis se préoccupant davantage de comprendre son évolution, pour revenir ensuite vers la préoccupation antérieure.

En Europe occidentale, les progrès des sciences naturelles, à l'époque classique, avaient inspiré, au lendemain d'un Moyen Age théologique, une recherche historique utilisant les méthodes des sciences nouvelles et recherchant dans le passé les répétitions et permanences qui auraient permis de former un corps de lois scientifique du comportement humain.

Voltaire, au siècle des Lumières, inspira la naissance de l'histoire des civilisations qui introduisait la préoccupation nouvelle de saisir globalement, en un tout continu, le destin de vastes collectivités se distinguant les unes des autres par des traits culturels propres. Le $\mathrm{XIX}^{\mathrm{e}}$ siècle engendra Karl Marx et ceux qu'on appela les grands historiens romantiques. Pour ceux-ci, l'histoire était une démarche intellectuelle par laquelle on pouvait arriver, à l'aide d'hypothèses compréhensives, à expliquer l'évolution humaine, à saisir le sens de l'histoire et, en dernière analyse, à se situer dans son mouvement.

Mais, bientôt, durant la deuxième moitié du siècle, se développait la sociologie qui, par sa prétention au positivisme, cherchait, comme autrefois l'histoire, à utiliser des méthodes calquées sur celles des sciences naturelles. Le positivisme des sciences sociales en France, l'empirisme en Angleterre, inspiraient les travaux historiques des Seignobos et des Acton, pour ne citer que ceux-là.

Cependant, à la même époque, surgissait d'Allemagne la philosophie de l'histoire qui devait provoquer le retournement du pendule par les grands travaux de ce que l'on appela l'école historique allemande. L'histoire redevenait compréhensive.

En Italie, Benedetto Croce approfondissait la pensée allemande et inspirait la recherche historique dans tout l'Occident. Pour lui, comme il le disait, "toute histoire est histoire contemporaine", voulant signifier par là, d'une part que le présent est lourd du passé, d'autre part et de façon beaucoup plus profonde, que le présent suggère à l'historien les questions, surgies des préoccupations de la société dans laquelle il vit, qu'il pose 
au passé. En France, de grandes revues devinrent le foyer des attitudes nouvelles: la Revue de Synthèse, d'abord, puis, les Annales. Dans tous les pays d'Occident, les historiens marxistes poursuivaient les grandes interrogations de Marx.

Science du passé, science du présent : voilà comment peut s'exprimer autrement l'opposition entre connaissance positive et connaissance compréhensive. Vouloir tout connaître de l'histoire, c'est prétendre se situer dans le passé pour lui-même, tandis que vouloir comprendre l'histoire, c'est analyser le passé à la lumière de la compréhension que l'on a du présent.

Les sciences sociales, parmi lesquelles la sociologie elle-même, se sont détachées au $\mathrm{XX}^{\mathrm{e}}$ siècle de leur proximité des sciences naturelles et ont développé des outils d'analyse particuliers, mieux appropriés à la connaissance de la réalité humaine. L'histoire compréhensive, après beaucoup d'hésitations, vit qu'elle pouvait utiliser ces outils d'analyse comme instruments d'objectivation des questions qu'elle allait dorénavant poser au passé. D'une part, les sciences sociales allaient suggérer aux historiens des interrogations nouvelles. D'autre part, grâce au bagage conceptuel dont elles se dotaient, les sciences sociales allaient permettre aux historiens de se défaire de ce qui jusqu'alors avait pu être des préjugés, des notions vagues et inconscientes. A l'avenir, les questions posées au passé seraient formulées dans les termes d'hypothèses scientifiques de travail.

Qu'on me permette de ne pas insister davantage sur la fécondité des méthodes nouvelles et sur l'enrichissement qu'en a reçu l'histoire. J'aimerais plutôt proposer une réflexion sur ce qu'il m'apparaît devoir être bientôt un nouveau positivisme en histoire, inspiré, encore une fois, des derniers développements des sciences sociales. Aujourd'hui, déjà, on ne parle presque plus, dans les sciences humaines, que de méthodes quantitatives, de mathématiques, de modèles, d'informatique, d'ordinateur, de langage Fortran, et de quoi encore? Bien sûr est-ce un avantage de pouvoir utiliser un langage rigoureux et de mesurer correctement. Mais dans la mesure où les historiens sont entraînés par cette préoccupation de tout formaliser et de tout connaître, l'histoire trouverait, dans les derniers développements des sciences humaines, le principe même d'un nouveau retournement du pendule. L'histoire risquerait d'être entraînée vers un nouveau positivisme.

Certes, l'inerte qui entraîne encore le pendule empêche que soit nettement entrevue l'esquisse du retournement. Mais déjà 
une tendance nouvelle se dessine dans le détachement de disciplines nouvelles du corps des sciences historiques. J'en prendrai pour preuve uniquement l'histoire économique, la "nouvelle" histoire économique, comme on dit déjà, et la démographie historique. Tout chiffrer, tout compter, tout mesurer, tout introduire dans l'ordinateur pour donner à l'information toutes les manipulations statistiques et mécanographiques possibles: voilà les préoccupations nouvelles. Ces disciplines veulent déjà voler de leur propres ailes, se détacher du corps de l'histoire et se rapprocher de celles des sciences sociales qui leur sont le plus étroitement apparentées.

Malgré les avantages certains de la connaissance plus étendue et de la précision supérieure, je vois deux difficultés aux tendances nouvelles. La première tient au fait que l'histoire, comme discipline, n'a pas de langage technique propre, semblable à ceux des autres sciences sociales, et n'a pas développé, comme l'ont déjà fait ses filles, un appareil de concepts et de catégories scientifiques. A ce titre, elle est à la merci des sciences nouvelles. Certes, la difficulté serait-elle moindre si les sciences sociales avaient déjà atteint une étape plus avancée de leur évolution et qu'elles possédaient déjà un corps d'instruments d'analyse bien rodés. Chacun sait qu'il n'en est rien. Les sciences sociales ont des outils imparfaits qu'elles discutent, qu'elles remettent sans cesse en question, qu'elles améliorent sans arrêt. Les transformations mêmes de la réalité sociale leur imposent de développer sans cesse de nouveaux instruments. A ce titre, l'histoire se place à la remorque des sciences sociales et risque, par là, d'être constamment en retard sur elles.

Pour ne parler que d'histoire économique, qui est mon domaine, combien d'historiens, inspirés par les théories économiques qui avaient cours durant la grande dépression des années ' 30 , se sont adonnés à des travaux extraordinaires d'histoire des prix, que certains ont poursuivi encore au-delà de la deuxième guerre mondiale, mais complètement détachés des préoccupations originelles ! Combien d'historiens s'adonnent, aujourd'hui, au calcul minutieux des comptabilités nationales, sans être parfaitement conscients des préoccupations théoriques des économistes! Quelle mode extraordinaire a acquise, chez les historiens, le mot modèle, sans qu'on prenne toujours bien garde qu'il implique, chez les économistes, qui en ont lancé la vogue, des méthodes très rigoureuses d'analyse statistique !

Quelle faiblesse, encore, est celle de l'histoire, devant les significations multiples que donnent à diverses notions abstraites 
les différentes sciences sociales, chacune s'enfermant parfois dans l'hermétisme de son langage propre ! Structure et conjoncture, voilà deux mots qui reçoivent des significations fort diverses selon que j'interroge les économistes, les sociologues, les anthropologues ou les linguistes. Dans une monographie que j'ai préparée sur la vie d'un entrepreneur canadien, Thomas Molson, j'ai dû longuement discuter, dans un chapitre d'introduction, la notion même d'entrepreneur. Car, interrogeant tour à tour économistes, sociologues et psychologues, j'ai trouvé une telle diversité et une telle hétérogénéité des définitions que j'ai dû me résoudre à reconnaître qu'il était impossible de trouver dans cette notion une catégorie typologique capable de suggérer une définition cohérente et rigoureuse.

Peut-être, finalement, serait-ce une des responsabilités de la science historique que de dénoncer chez ses filles, les sciences sociales, leurs légèretés d'adolescence. Il lui reviendrait ainsi une contribution active à l'édification d'une science sociale intégréé, si tant est qư'il soit pôssible et soühaitable d'ériger un tel monument.

La deuxième difficulté que je vois devant les tentatives actuelles de traduire toute information en langage d'ordinateur réside dans le risque de voir, sinon disparaître, du moins diminuer une fonction indispensable de l'historien: celle, dans son enquête de sélectionner les faits et d'arrêter son choix sur ceux qu'il considère significatifs. La machine ne reçoit que l'information qui lui est offerte en catégorie définissant des fréquences at des corrélations et moyennant la traduction de cette information en un langage approprié. A ce titre, on aurait tendance à confier au programmeur de l'ordinateur une des tâches fondamentales de l'historien.

Pourtant, les historiens se sont accoutumés, de toute antiquité, à choisir, dans la masse indifférenciée des faits et des événements du passé, ceux qui leur paraissent dignes de leur attention, ceux auxquels ils trouvent et donnent signification, ceux qui, dorénavant, recevront, par la lumière qu'ils auront dirigée sur eux, la qualité insigne de faits historiques. Edward Hallett Carr disait, à ce titre, que l'historien fait l'histoire tout autant, sinon davantage, que les acteurs de l'histoire eux-mêmes. Un homme politique, à qui je m'adressais un jour pour discuter avec lui de la préparation d'un ouvrage sur des événements auxquels il avait participé, termina l'entretien en me lançant: "Monsieur, vous écrivez l'histoire, nous, nous la faisons." J'avoue que, sans aucune prétention et bien humblement, je prenais 
plaisir, dans mon for intérieur, à me remérorer la page magnifique de Carr.

L'histoire, même à titre de discipline de la connaissance, n'est pas que descriptive; elle est, d'abord et avant tout, compréhensive. Elle ne donnera jamais de réponse qu'aux questions qui seront posées. La richesse de l'histoire tient à l'intelligence des questions que les historiens n'ont eu de cesse de poser au passé. Nul programmeur, avec toute la compétence voulue pour nourrir d'informations l'ordinateur le plus avancé technologiquement, n'arrivera jamais à se substituer à l'historien.

Loin de moi le refus des méthodes les plus modernes et des outils les plus efficaces de la connaissance de l'homme ! J'en fais moi-même suffisamment usage pour ne pas les renier. J'ai voulu simplement éveiller votre attention à cette tentation du positivisme qui entraîne aujourd'hui de nouveau l'histoire à la suite du développement, par les sciences sociales, de nouvelles méthodes de recherche.

L'histoire, comme les sciences sociales, est connaissance de l'homme. Mais, à cause de la richesse des outils d'analyse que ces dernières ont développés, elle est, en quelque sorte, à leur remorque. Car elle ne procède pas par catégories scientifiques. Mais elle procède autrement. L'histoire en effet n'est pas que connaissance.

\section{L'histoire, comme culture}

L'histoire est, en effet, prise de conscience par l'homme du processus d'évolution qui l'entraîne en même temps qu'introduction de l'homme à l'intérieur de ce processus. A la vérité, si l'histoire-connaissance ne peut jamais se résoudre à devenir une discipline positive et que, sans cesse, elle est ramenée vers la compréhension du processus d'évolution, c'est parce qu'elle est l'objet même et l'instrument tout à la fois de l'interrogation de l'homme. Il n'est pas sans signification que le mot histoire désigne à la fois l'objet de l'enquête et la méthode qui sert à percevoir cet objet. Par le même mot, le langage désigne le passé et l'interrogation de l'homme sur son passé.

Comme culture, l'histoire est à la fois perception du relief du passé et élargissement de la dimension du présent. Par la prise en considération du relief du passé, plutôt que de son uniformité, l'histoire permet à la conscience de l'homme, convaincue dorénavant du continu de son évolution, de joindre le passé au présent et, par la profondeur entrevue, de se projeter dans 
l'avenir. Il n'y a pas de demain, sans hier; pas de prospective, sans rétrospective. L'histoire permet à l'humanité de s'appuyer sur l'acquis de la civilisation, dans le mouvement même de sa projection dans le futur.

L'histoire-culture est, aussi, élargissement de la dimension du présent. L'homme découpe le temps en unités qui marquent les dimensions diverses du présent dans lequel il veut se situer. "Cet instant", "aujourd'hui", "cette année", "le XX siècle", "la civilisation occidentale", "l'histoire humaine": voilà des expressions qui marquent des présents différents.

Le 20 juillet 1969, quand le premier terrien mit pied sur la lune, le temps entra dans la conscience de l'homme sous une nouvelle dimension: la dimension cosmique. Le cosmos ne sera jamais plus une projection imaginative, comme il l'était demeuré trop longtemps depuis que l'homme interrogeait le ciel étoilé. Au moment même où l'homme se tire de l'attraction terrestre pour entrer dans le cosmos, celui-ci, par un effet dialectique, pénètre dans la conscience de i'homme pour le transformer. Etendre ies dimensions du présent c'est, pour l'homme, se projeter, à travers l'histoire, aux confins les plus éloignés de ses possibilités, étendre l'aire de son activité et de sa créativité et, tout en même temps, se projeter dans l'avenir.

On aura saisi comment sont indissociablement liées les deux notions de temps et d'espace. Etendre les dimensions du présent, c'est, pour l'homme, étendre les dimensions de l'espace dans lequel il évolue. Dans la conscience, la perception de l'une ne va pas sans l'autre. Car, ensemble, elles définissent l'aire à l'intérieur de laquelle l'homme tisse ses liens de solidarité. Déjà, par sa hâte de capter les ondes qu'auraient pu émettre des êtres vivants sur d'autres planètes, l'homme offre de lancer des liens nouveaux dans le cosmos.

A n'en pas douter, l'histoire est culture. C'est par elle que l'homme éveille sa conscience à lui-même. Le philosophe allemand, Dilthey, voyait en elle la même utilité pour l'humanité que celle de l'introspection pour l'individu. De même que celui-ci par l'introspection prend conscience de lui-même, de ses lignes de force comme de ses limites et oriente son action, ainsi l'humanité par l'histoire se révèle à elle-même, prend conscience de son évolution et se projette dans l'avenir.

Ainsi donc, l'histoire est, à la fois connaissance et culture. Grâce à cette double dimension, elle participe de plein droit aux 
sciences humaines. Certes, ne possède-t-elle pas des instruments de connaissance et des outils d'analyse aussi efficaces que ceux qu'ont fabriqués les sciences nouvelles. Mais elle a déjà appris à les leur emprunter. Toutefois, elle a développé de toute antiquité, une interrogation sur elle-même qui lui permet de se remettre en question constamment. A ce titre elle est beaucoup plus riche que plusieurs sciences sociales dont le formalisme n'a d'égal que la pauvreté épistémologique. Ainsi l'histoire joue-t-elle un rôle positif dans le concert des sciences sociales que nulle ne saura lui ravir.

Au contraire des autres disciplines qui cherchent à marquer l'indépendance obligée du sujet connaissant et de l'objet d'analyse, l'histoire établit constamment les ponts qui relient entre eux, dans une interdépendance étroite, les deux pôles de l'opposition épistémologique. L'homme, en tant qu'objet d'étude, devient mieux connu et cette connaissance reflète sur lui-même en tant que sujet-connaissant, pénètre sa conscience, le transforme et entraîne sa marche en avant. Dans la même démarche intellectuelle, connaissance et conscience se nourrissent réciproquement l'une l'autre et inspirent les projets de l'homme. A ce titre, Collingwood avait raison de prétendre qu'il n'y a d'histoire qu'histoire de l'esprit.

L'homme n'aura de cesse de poser des interrogations nouvelles sur lui-même. Elargissant toujours le champ de sa conscience, des choix nouveaux s'imposeront à son action.

Mais le problème du choix est responsable de la présence inéluctable des valeurs en science humaine. Si j'appelle idéologie l'ensemble des valeurs qui motivent mes choix, il me devient interdit de me soustraire à l'obligation de reconnaître le rôle indispensable de l'idéologie dans les sciences humaines.

Certes, je concède immédiatement qu'il faille introduire une distinction fondamentale entre valeurs de l'action et valeurs de la connaissance. Celles qui motivent mes choix politiques ne sont pas du même ordre que celles qui inspirent mes hypothèses de travail. Bien sûr, il faut s'attendre à ce que la distance entre les deux niveaux de valeurs ne soit pas trop grande chez le citoyen-homme de science qui recherche la cohérence de sa personnalité et l'honnêteté envers lui-même. A preuve, cependant, que j'ai raison de distinguer les deux ordres, c'est qu'il est tellement fréquent de ne pas rencontrer cette exigence spirituelle et qu'il y ait si souvent écart, voire même opposition, chez le même individu, entre ses valeurs d'homme de science et ses valeurs de citoyen. 
Les sciences humaines, obligées qu'elles sont de sans cesse valider leurs interrogations, de remettre en question leurs hypothèses de travail, d'affiner constamment leurs outils d'analyse, ne peuvent en aucun temps prétendre se passer d'idéologie. Toute hypothèse de travail, en sciences humaines, est lourde d'un contenu de valeurs. Sans valeur, il devient impossible d'exercer son esprit critique sur l'efficacité des instruments qu'on utilise, il devient même impossible de proposer quelqu'hypothèse de travail que ce soit. Au vrai, l'idéologie est, pour les sciences humaines, ce qu'est l'imagination créatrice pour les mathématiques.

Celui qui prétend être à l'abri de toute idéologie refuse de remettre en question son interrogation scientifique et refuse en même temps de reconnaître les valeurs que chacun porte en soi-même. Il se trompe lui-même et trompe les autres. Au fait, la thèse de la fin des idéologies est la plus idéologique des thèses qui soient. Car, interdisant l'exercice de tout esprit critique, elle s'oppose aux développements de la science et, débouchant sur le plan de l'action politique, devicnt la défense la plus insidieuse et par là, la plus efficace, de l'ordre établi.

En l'absence des valeurs qui inspirent les hypothèses de travail, les sciences humaines seraient totalement incapables de se développer. L'idéologie est le ferment de la connaissance.

Par sa double orientation vers la connaissance et la culture, l'histoire est un catalyseur dans l'évolution des sciences humaines. Forçant l'homme à nourrir sa conscience des connaissances plus riches qu'il acquiert de lui-même grâce aux sciences humaines, l'histoire suggère à l'homme les orientations à prendre dans sa quête de liberté et de solidarité et, aux disciplines scientifiques, les interrogations nécessaires à leur développement.

S'il n'est pas prétentieux, pour un historien d'affirmer que l'histoire a engendré les sciences humaines, il ne l'est pas davantage de proposer qu'elle ne cesse de les nourrir. Autant demain qu'aujourd'hui, les sciences humaines devront s'accommoder de ce personnage encombrant qui demeure au carrefour de leurs préoccupations. 